This is an electronic reprint of the original article. This reprint may differ from the original in pagination and typographic detail.

Author(s): Burger, Birgitta; Saarikallio, Suvi; Luck, Geoff; Thompson, Marc; Toiviainen, Petri

Title: $\quad$ Relationships between perceived emotions in music and music-induced movement

Year: $\quad 2013$

Version:

Please cite the original version:

Burger, B., Saarikallio, S., Luck, G., Thompson, M., \& Toiviainen, P. (2013).

Relationships between perceived emotions in music and music-induced movement.

Music Perception, 30(5), 517-533. https://doi.org/10.1525/MP.2013.30.5.517

All material supplied via JYX is protected by copyright and other intellectual property rights, and duplication or sale of all or part of any of the repository collections is not permitted, except that material may be duplicated by you for your research use or educational purposes in electronic or print form. You must obtain permission for any other use. Electronic or print copies may not be offered, whether for sale or otherwise to anyone who is not an authorised user. 


\section{Relationships Between Perceived Emotions in Music and Music-induced Movement}

Birgitta Burger, Suvi Saarikallio, Geoff Luck, Marc R. Thompson, \& Petri Toiviainen University of Jyväskylä, Jyväskylä, Finland

LISTENING TO MUSIC MAKES US MOVE IN VARIOUS ways. Several factors can affect the characteristics of these movements, including individual factors and musical features. Additionally, music-induced movement may also be shaped by the emotional content of the music, since emotions are an important element of musical expression. This study investigates possible relationships between emotional characteristics of music and music-induced, quasi-spontaneous movement. We recorded music-induced movement of 60 individuals, and computationally extracted features from the movement data. Additionally, the emotional content of the stimuli was assessed in a perceptual experiment. A subsequent correlational analysis revealed characteristic movement features for each emotion, suggesting that the body reflects emotional qualities of music. The results show similarities to movements of professional musicians and dancers, and to emotion-specific nonverbal behavior in general, and could furthermore be linked to notions of embodied music cognition. The valence and arousal ratings were subsequently projected onto polar coordinates to further investigate connections between the emotions of Russell's (1980) circumplex models and the movement features

Received: May 4, 2012, accepted January 18, 2013.

Key words: music, music-induced movement, motion capture, emotion, circumplex model of affect

$\mathrm{L}$ ISTENING TO MUSIC MAKES US MOVE IN VARIous ways (Leman, 2007; Leman \& Godøy, 2010). Several factors can affect the characteristics of these movements: Individual factors such as personality and preference have been found to influence movability to music (Luck, Saarikallio, Burger, Thompson, \& Toiviainen, 2010), as have music-intrinsic features such as beat strength and pulse clarity (Burger, Thompson,
Saarikallio, Luck, \& Toiviainen, 2010; Van Dyck et al., 2010). Besides such factors, music-induced movement may be shaped by the emotional content of the music. Emotions are an essential component of musical expression (e.g., Gabrielsson \& Lindström, 2001) that can have a strong influence, for instance, on the listener's mood (e.g., Saarikallio, 2011). However, only a few studies have addressed the connection between emotions and music-induced movement.

Research on emotion-specific nonverbal behavior has nonetheless shown that it is possible to successfully express and communicate emotional states to observers through body movements. Already in 1872, Darwin assigned certain body movements and postures quite specifically to emotional states; joyful movements, for example, were described as jumping, stamping, body thrown backwards and shaking, and upright torso and head, whereas anger was characterized by trembling body, shaking fist, erected head, and expanded chest. Sad movements were described as passive, motionless, and a downward directed head. Despite these finding, the topic was rather neglected for a while-probably due to lack of appropriate technologies-and research focused more on facial expression (e.g., Ekman, 1982) following the assumption that gross body movements would only communicate intensity of emotions, but not qualitative characteristics (Ekman \& Friesen, 1974).

Nevertheless, several recent studies have shown that emotions can be expressed by, and successfully recognized from, body postures or movements. From the early seventies onwards, Clynes $(1980,1992)$ investigated in the context of human-machine interaction how communication can be made more human-like and more efficient. For this purpose he developed a repertoire of so-called dynamic transient forms, or sentic forms that are specific for each emotion.

Wallbott (1998) conducted a study in which he used a scenario-based approach, with professional actors performing certain emotions. Besides a recognition test, he also analyzed movement features characteristic and distinctive for each emotion category; for instance, sadness was expressed with a collapsed body, whereas joy and anger were associated with a more upright torso. Anger was the most active emotion, followed

Music Perception, volume 30, issue 5, pp. 517-533, issn 0730-7829, electronic issn 1533-8312. C 2013 by the Regents of the university of California all RIGHTS RESERVED. PLEASE DIRECT ALL REQUESTS FOR PERMISSION TO PHOTOCOPY OR REPRODUCE ARTICLE CONTENT THROUGH THE UNIVERSITY OF CALIFORNIA PRESS'S RIGHTS AND PERMISSIONS WEBSITE, HTTP://WWW.UCPRESSJOURNALS.COM/REPRINTINFO.ASP. DOI: 10.1525/MP.2013.30.5.517 
by joy and sadness. Lateral arm movement and dynamics/ power were mostly related to anger, less for joy, and even less for sadness. Spatially expansive movements were used to express anger and joy, whereas sad movements covered less space.

Dael, Mortillaro, and Scherer (2012) also used actors in a scenario-based approach, and manually labeled their data with a self-developed microcoding system (Body Action and Posture system; BAP) including 49 movement variables, such as "shoulder action" or "arm action towards body." Their analysis revealed that angry movements were characterized by forward movement, joy was communicated with repetitive hand and symmetric arm movement, pleasure was characterized by the head tilted up and asymmetrical arm movement, whereas sad movements were characterized by both arms at rest and down.

A different approach was chosen by De Meijer (1989), who used actors performing several movement characteristics instead of emotions. These movements differed in general features, such as trunk or arm movement, velocity, and spatial direction. In a subsequent step, observers attributed emotional characteristics to the movements. The results suggested that fast, active, open, light, and upward directed movements with raised arms and a stretched trunk were perceived as happy, whereas strong and fast movements with a bowed torso and a high force were perceived as angry. In contrast, sad movements were described as slow, light, downward directed, with arms close to the body.

Coulson (2004) used pictures of static body postures of computer-generated figures, which he manipulated systematically in several parameters. He found that a forward bended and directed body with hands in front of the chest and bent elbows was associated with anger, whereas a forward leaning and downwards bent/ crouched body with downwards directed hands was characterized as sad, and a backwards bent body with upraised hands and arms was associated with happiness.

Furthermore, emotions are a central element of music and have been investigated in a large number of musicrelated studies. According to Krumhansl (2002), people report that their primary motivation for listening to music is its emotional impact. Various rating experiments have shown that listeners are able to perceive emotional content in music. One of the issues that researchers in this field face concerns the choice of emotion categories and models that can cover musical emotions successfully. As Eerola and Vuoskoski (2011) noted, there are basically three different approaches that have been used in studies on music and perceived emotions: discrete (basic) emotions (e.g., Balkwill \&
Thompson, 1999; Gabrielsson \& Juslin, 1996), domainspecific emotion models, such as GEMS (Geneva Emotion Music Scale, see Zentner, Grandjean, \& Scherer, 2008), and dimensional models (e.g., Ilie \& Thompson, 2006; Schubert, 1999). The discrete model describes emotions as unidimensional, independent from each other, and derived from a limited number of universal and innate basic emotions, such as happiness, anger, sadness, or fear. The advantage of using this approach is the possibility to focus on emotions that are commonly expressed and represented by music, and name them in clear terms. Inspired by this approach, domain-specific discrete scales such as GEMS have been developed to create a repertoire of emotion terms that is highly music-specific. A disadvantage of discrete emotions is, according to Eerola and Vuoskoski (2011), that they can be mixed and confused with each other in discrimination tasks. This issue might be reduced by using a dimensional approach, in which all affective states are represented as a combination of two (usually valence and arousal) or three (valence, arousal, and tension) mutually independent dimensions. A common way to illustrate dimensional models is in a spatial design, such as a circle, with two bipolar dimensions-valence forming the horizontal axis, and arousal the vertical axis (in case of a three-dimensional model, tension would form the third axis resulting in a spherical design). Examples of such approaches are the circumplex models of affect by Russell (1980) and Schlosberg (1954). Eerola and Vuoskoski (2011) compared the different models and showed that, although both dimensional and discrete models performed similarly, however, the discrete model performed worse when it came to ambiguous emotions. Additionally, they found that a twodimensional model consisting of valence and arousal successfully represented music-related emotions.

Musical emotions are conveyed not only by the music itself, but also through movement. While movements are required, for example, to produce sounds when playing a musical instrument, studies have shown that there are certain additional movements that are not used for the actual sound production, but for conveying emotions and expressivity (e.g., Wanderley, Vines, Middleton, McKay, \& Hatch, 2005). Dahl and Friberg (2007) investigated a marimba, a bassoon, and a saxophone player, who each performed a piece of music with different emotional intentions (happy, angry, sad, and fearful). Observers could, when presented with only visual elements of the performance, detect the happy, angry, and sad performances, though failed for the fearful performances. In a second task, they were 
asked to identify movement cues used to convey the different emotions. Happy intention was found to be communicated by medium regularity and fluency, high speed, and high amount of movement of the whole body, whereas sad intention was conveyed by very regular and fluent movement, low in speed and quantity. Angry intention was communicated with medium regularity, low fluency, high speed, and somewhat high amount of movement. Fearful intention was perceived as being characterized by medium regular and fluid, rather fast, and rather small amounts of movement. Burger and Bresin (2010) developed a small robot displaying different emotions based on the movement cues found by Dahl and Friberg (2007) and used, for example, large, fluent, and circular movements to convey happiness, large, irregular, and jerky movements for anger, and slow, regular, and reduced movements to convey sadness. In a perceptual experiment, all emotions were successfully recognized by the observers.

More direct links between music and emotionspecific movement have been investigated in research on dance, in which movement is the only way to convey expressivity and emotion. Camurri, Lagerlöf, and Volpe (2003) describe a study in which professional dancers were asked to perform the same dance with four different emotional intentions. In a qualitative analysis, they identified characteristic movements for the expression of these emotions; anger was, for instance, characterized by short movements, frequent tempo changes, short stops between changes, and dynamic and tense movement, whereas happiness was associated with frequent tempo changes, longer stops between changes, dynamic movement, and changes between low and high tension. Sadness was portrayed by long and smooth movements, few tempo changes, and low tension, whereas fear was represented by frequent tempo changes, long stops between changes, high tension, and movements close the center of the body.

Camurri et al. (2003) and Camurri, Mazzarino, Ricchetti, Timmers, and Volpe (2004) developed a video-based analysis tool to recognize and classify expressive gestures in professional dance performances. This tool was used, for example, in Castellano, Villalba, and Camurri (2007), who studied dynamic qualities of motion cues as opposite to different movement shapes that might convey emotional qualities. They recorded the same gesture performed in four emotions (happy, angry, sad, and pleasurable) and could identify two main movement characteristics that differentiated between them: Quantity of Motion (QoM), defined as "the overall measure of the amount of detected motion, involving velocity and force" (p. 47), and Contraction
Index (CI), defined as "the contraction and expansion of the body [...], i.e., the minimum rectangle surrounding the body" (p. 47) in a two-dimensional space. CoM could discriminate between high and low arousal (anger and joy being associated with high QoM, and sadness and pleasure with low QoM), while CI could distinguish between positive and negative emotions (joy and pleasure with low CI vs. anger and sadness with high CI).

Furthermore, Boone and Cunningham (1998) reported results of a dance study in which actors displayed basic emotions. They found that angry movements were associated with a great number of directional changes of the torso, as well as tempo changes, whereas happy movements were characterized by upward arm movements away from the torso. Sad movements were portrayed with downward gaze, low muscle tension, and a slackened body, and fearful movements were described as more rigid with a head-up "alert" posture.

Besides being an important cue in music performance and professional dance, movement also plays a considerable role in every-day music behavior. Keller and Rieger (2009), for example, stated that simply listening to music can induce movement, and in a self-report study conducted by Lesaffre et al. (2008), most participants reported moving when listening to music. Janata, Tomic, and Haberman (2011) reported in a study on groove that the wish to move along with the music is related to positive affect. They furthermore asked participants to tap to the music and found that, with increasing groove in the music, participants not only moved the finger/hand, but also other body parts, such as feet and head. Additionally, the tapping condition (isochronous versus free tapping) influenced the amount of movement: the more "natural" the tapping condition, the more movement was exhibited.

In general, people tend to move to music in an organized way; for example, by mimicking instrumentalists' gestures or rhythmically synchronizing with the pulse of the music by tapping the foot, nodding the head, or moving the whole body in various manners (Leman \& Godøy, 2010). Moreover, Leman (2007) suggests, "Spontaneous movements [to music] may be closely related to predictions of local bursts of energy in the musical audio stream, in particular to the beat and the rhythm patterns" (p. 96). Such utilization of the body is the core concept of embodied cognition, which claims that the body is involved in or even required for cognitive processes (e.g., Lakoff \& Johnson, 1980, 1999, or Varela, Thompson, \& Rosch, 1991). Human cognition is thus highly influenced by the interaction and interplay of mind/brain, sensorimotor capabilities, body, and environment, and determined by the body with 
its specific shape, including particular perceptual and motor capabilities. Following this, we can approach music (or musical involvement) by linking our perception of it to our body movement (Leman, 2007). One could postulate that our bodily movements might reflect, imitate, help parse, or support the understanding of the content of music, be it musical features, such as beat, rhythm, melody, or tonality, or emotional characteristics. Leman suggests that corporeal articulations could be influenced by three (coexisting) components or concepts: Synchronization, Embodied Attuning, and Empathy, which differ in the degree of musical involvement and the kind of action-perception couplings. Synchronization forms the fundamental component, as synchronizing to a beat is easy, spontaneous, and possible even when paying minimal attention to the music. The beat serves as the basic musical element from which more complex structures emerge. Leman thus suggests the term inductive resonance, which refers to more active control, imitation, and prediction of movements to display beat-related features in the music (the opposite of passively tapping to a beat) as the first step in engaging with the music. The second component, Embodied Attuning, concerns the linkage of body movement to musical features more complex than the basic beat, such as melody, harmony, rhythm, tonality, or timbre. Following this idea, movement could be used to reflect and imitate the musical structure in order to understand it. Finally, Empathy is seen as the component that links music, or rather musical features, with expressivity and emotions. In other words, the listener feels and identifies with the emotions expressed in the music and imitates and reflects them by using body movement.

The aim of this study was to investigate relationships between the emotional content of music and characteristics of music-induced, quasi-spontaneous movement. We first conducted a motion-capture experiment (Experiment 1) and computationally extracted various movement characteristics from these data. Additionally, the perceived emotional content of the music used in the first part was gathered in a rating experiment (Experiment 2). Based on the considerations mentioned above, we chose to represent the emotional content with a two-dimensional model containing valence and arousal, and the four basic emotions happiness, anger, sadness, and tenderness (corresponding to the four quadrants of the dimensional model). We restricted this study to the use of perceived emotions as opposed to felt emotions, since previous literature has mostly focused on the former. It is important to distinguish between perceived and felt emotions, as these two kinds of responses have been found to differ from each other (e.g., Evans \& Schubert, 2008; Gabrielsson, 2002).

In this study we were mainly interested in two aspects. First, we investigated whether movement and perceived emotional content are related, and more particularly, which body parts and movement characteristics can be associated with different emotions expressed in music. Second, we attempted to link our results to Leman's (2007) framework on corporeal articulations.

Based on the studies mentioned above (emotionspecific nonverbal behavior, music performance, and professional dance), we predicted that different emotions would be associated with different combinations of movement features as follows:

1. Music perceived as active would result in a high amount of movement occupying a large area, as active music might be encouraging listeners to move.

2. Active emotions, such as happiness and anger, would show similar patterns, with smoother movements observed for happy music than for angry music.

3. Inactive music, including sad and tender music, would result in a small amount of movement occupying a small area, as inactive music is less movementstimulating than active music.

4. Happy, and probably pleasant, music would be related to body rotation (around the vertical axis).

5. Active emotions, such as happiness and anger, would lead to a more upward directed and stretched torso, whereas for inactive emotions (sadness and tenderness), the torso would be more directed forwards.

6. Happy (and pleasant) stimuli would be related to large hand distance.

Based on the first four predictions, we further assumed that the overall movement complexity would increase with active (especially happy) music and decrease with inactive music (especially sad) music, since active music is more movement stimulating than inactive music. To this end, we developed a feature that operationalizes the overall complexity of full-body movement.

\section{Method}

The data used in this study were obtained in two consecutive experiments. In Experiment 1, music-induced movement data were collected. In Experiment 2, the (perceived) emotional content of the stimuli used in Experiment 1 was assessed.

EXPERIMENT 1 - MOVEMENT TASK

Participants. A total of 60 participants took part in Experiment 1 (43 females; age range $=19-32, M=24.0$, 
(a)
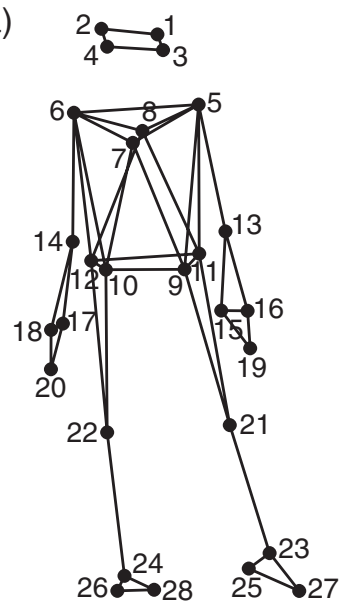

(b)

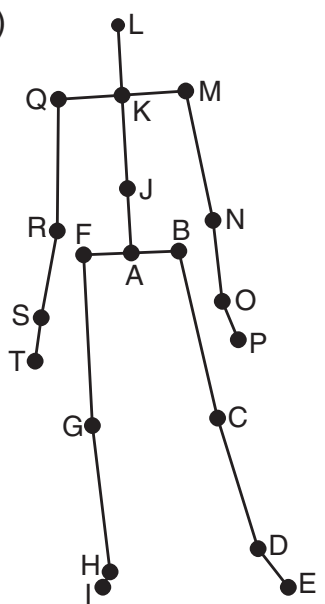

FIGURE 1. (a) Anterior view of the location of the markers attached to the participants' bodies; (b) Anterior view of the locations of the secondary markers/joints used in the analysis.

$S D=3.3$ ). Six participants had received formal music education, and three participants were dance pedagogues. Participants were rewarded with a movie ticket.

Stimuli. Participants were presented with 30 randomly ordered musical stimuli representing the following popular music genres: Techno, Pop, Rock, Latin, Funk, and Jazz. All stimuli were naturalistic (existing pop music) to make the experiment as ecologically valid as possible, and had a uniform emotional content throughout. Participants were on average unfamiliar with every stimulus. All stimuli were $30 \mathrm{~s}$ long, nonvocal, and in 4/4 time, but differed in their rhythmic complexity, pulse clarity, and tempo. An overview of the stimuli can be found in Appendix A.

Apparatus. Participants' movements were recorded using an eight-camera optical motion capture system (Qualisys ProReflex) tracking, at a frame rate of 120 $\mathrm{Hz}$, the three-dimensional positions of 28 reflective markers attached to each participant. The locations of the markers can be seen in Figure $1 \mathrm{a}$, and can be described as follows $(\mathrm{L}=$ left, $\mathrm{R}=$ right, $\mathrm{F}=$ front, $\mathrm{B}=$ back): 1: LF head; 2 : RF head; 3 : LB head; $4: \mathrm{RB}$ head; 5: L shoulder; 6: $\mathrm{R}$ shoulder; 7: breastbone; 8 : spine; 9: LF hip; 10: RF hip; 11: LB hip; 12: RB hip; 13: L elbow; 14: R elbow; 15: L wrist/radius; 16: L wrist/ulna; 17: $\mathrm{R}$ wrist/radius; 18: $\mathrm{R}$ wrist/ulna; 19: $\mathrm{L}$ middle finger; 20: $\mathrm{R}$ middle finger; 21 : $\mathrm{L}$ knee; 22: $\mathrm{R}$ knee; 23: L ankle; 24: $\mathrm{R}$ ankle; 25: $\mathrm{L}$ heel; 26: $\mathrm{R}$ heel; 27: $\mathrm{L}$ big toe; 28: $\mathrm{R}$ big toe. The musical stimuli were played back via a pair of Genelec 8030A loudspeakers using a Max/MSP patch running on an Apple computer.
Procedure. Participants were recorded individually and were asked to move to the stimuli in a way that felt natural. Additionally, they were encouraged to dance if they wanted to, but were requested to remain in the center of the capture space indicated by a $115 \times 200 \mathrm{~cm}$ carpet.

Dancing in a motion capture lab might appear as a very artificial situation, and it is expectable that participants felt strained and uneasy. However, the experimenters made sure that the participants felt as comfortable as possible and did not watch the participants during dancing, as they were concealed by the technical equipment. Most participants reported after the experiment that they enjoyed the experience and quickly forgot about the equipment and the experimenters. Only one participant felt so uncomfortable that she decided to discontinue the experiment. Therefore, we think that participants were able to fulfill the task without being much biased by the situation and environment.

Movement feature extraction. In order to extract various kinematic features, the MATLAB Motion Capture (MoCap) Toolbox (Toiviainen \& Burger, 2011) was used to first trim the data to the duration of each stimulus. To remove redundant data while retaining the essential body parts and joints, a set of 20 secondary markerssubsequently referred to as joints-was derived from the original 28 markers. The locations of these 20 joints are depicted in Figure 1b. The locations of joints C, D, E, G, $\mathrm{H}, \mathrm{I}, \mathrm{M}, \mathrm{N}, \mathrm{P}, \mathrm{Q}, \mathrm{R}$, and T are identical to the locations of one of the original markers, while the locations of the remaining joints were obtained by averaging the locations of two or more markers; joint A: midpoint of the four hip markers; B: midpoint of markers 9 and 11 (left hip); F: midpoint of markers 10 and 12 (right hip); J: midpoint of breastbone, spine, and the hip markers (midtorso); K: midpoint of shoulder markers (manubrium), L: midpoint of the four head markers (head); O: midpoint of the two left wrist markers (left wrist); S: midpoint of the two right wrist markers (right wrist). From the three-dimensional joint position data, the instantaneous velocity and acceleration were estimated using numerical differentiation based on the SavitzkyGolay smoothing FIR filter (Savitzky \& Golay, 1964) with a window length of seven samples and a polynomial order of two. These values were found to provide an optimal combination of precision and smoothness in the time derivatives. Thereafter, ten movement features were extracted from these data:

- Postural features:

- Torso Tilt: vertical tilt of the torso (Joints A-K), positive tilt being related to bending forward. 
- Hand Distance: distance between hands (Joints P and $\mathrm{T}$ ).

- Foot Distance: distance between feet (Joints (E and I).

- Local kinematics:

- Magnitude of Head Acceleration (Joint L).

- Magnitude of Hand Acceleration (Joints P and T).

- Magnitude of Foot Acceleration (Joints E and I).

- Global kinematics:

- Area of Movement: the smallest rectangle that contains the projection of the trajectory of the Center of Mass (Joint A) on the horizontal plane (i.e., floor), averaged across four-second analysis windows with a two-second overlap.

- Fluidity: overall movement fluidity/smoothness measure based on the ratio of velocity to acceleration. The combination of high velocity and low acceleration reflects fluid movement, whereas the combination of low velocity and high acceleration reflects non-fluid movement.

- Rotation Range: amount of rotation of the body (joints $\mathrm{M}$ and $\mathrm{Q}$ ) around the vertical axis.

- Movement Complexity: based on Principal Components Analysis (PCA) of the position data (all joints). PCA, in this case, can be understood as a decomposition into different (independent) movement components. The PCA was performed on individual subject level, i.e., one PCA per participant and stimulus. Subsequently, the cumulative sum of the proportion of (explained) variance contained in the first five PCs was determined. We found that for five PCs the cumulative variance had the highest range of variation across movement recordings, so using five PCs discriminated best between complex and non-complex movement. The proportion of variance explained with the first five PCs varied between $67.0 \%$ and $99.8 \%$, with an average of $85.5 \%$ (subject level). For an excerpt with $99.8 \%$ explained variance, almost all movement can be explained with the first five components, whereas for an excerpt with $67.0 \%$ explained variance, $33 \%$ cannot be explained by the first five components (and more components are needed). Thus, a high proportion of unexplained variance (i.e., a low cumulative sum) would mean that the underlying movement is complex, as a high number of PCs is needed to explain the movement sufficiently. A low proportion of unexplained variance (i.e., a high cumulative sum), on the other hand, implies a simpler movement, as it can be sufficiently explained with a low number of PCs. Figure 2 illustrates this feature by displaying projections of the first five principal components of complex and noncomplex movements.

Apart from the Area of Movement feature, all movement features were calculated based on a local coordinate system, in which joint $A$ was located at the origin, and segment $\mathrm{BF}$ was parallel to the $\mathrm{x}$-axis of the coordinate system.

Subsequently, the instantaneous values of each variable were averaged across the participants for each stimulus presentation. This yielded a total of ten statistical movement features for each of the 30 stimuli.

EXPERIMENT 2 - PERCEPTUAL TASK

Participants. Thirty-four Finnish musicology students participated in experiment 2 (17 females; age range $=$ $21-47, M=25.7, S D=5.9)$. All participants were familiar with concepts of music and emotion research, such as different emotion models and research on perceived and felt emotions. None of the participants of this experiment participated in Experiment 1.

Stimuli. The same 30 musical excerpts as in Experiment 1 were used. To conduct the experiment in a reasonable time, we shortened the stimuli to $15 \mathrm{~s}$ by removing the first and last $7.5 \mathrm{~s}$ of the original excerpt. As both the emotional content of the stimuli and the characteristics of the movement were stable throughout each stimulus (correlations between movement features calculated for 15- and 30-s excerpts and the perceptual ratings resulted in a very similar pattern), shortening the stimuli did not have a notable effect on the correlations between movement and perceptual features.

Procedure. The participants were randomly divided into two groups that were presented with the stimuli at the same time, but in two different random orders. Each stimulus was presented once, followed by a break of $40 \mathrm{~s}$. The participants' task was to rate each stimulus according to its emotional content on six different seven-point scales:

- Arousal: inactive - active

- Valence: unpleasant - pleasant

- Happiness: not happy - happy

- Sadness: not sad - sad

- Tenderness: not tender - tender

- Anger: not angry - angry

Participants were explicitly told in the instructions to rate the excerpts according to what they thought the music expresses/conveys and not what they personally felt when listening to the music. 
(a)
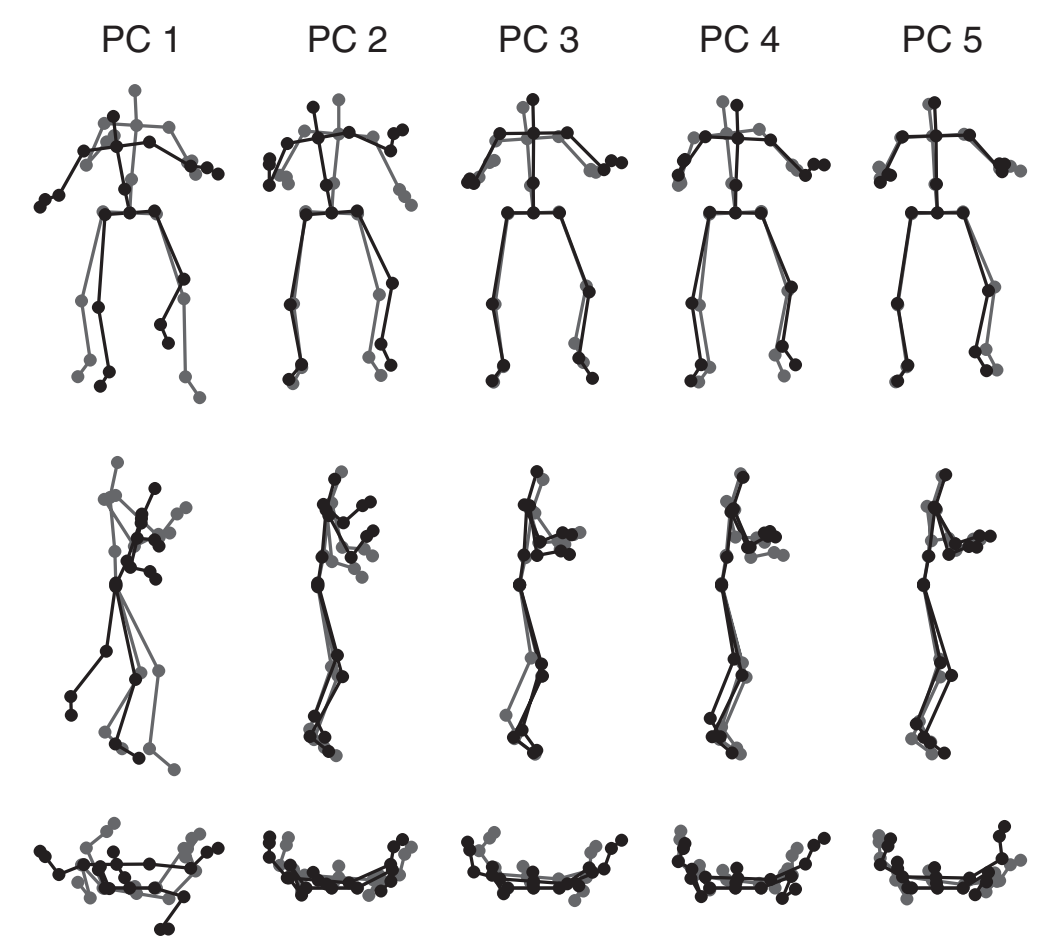

(b)
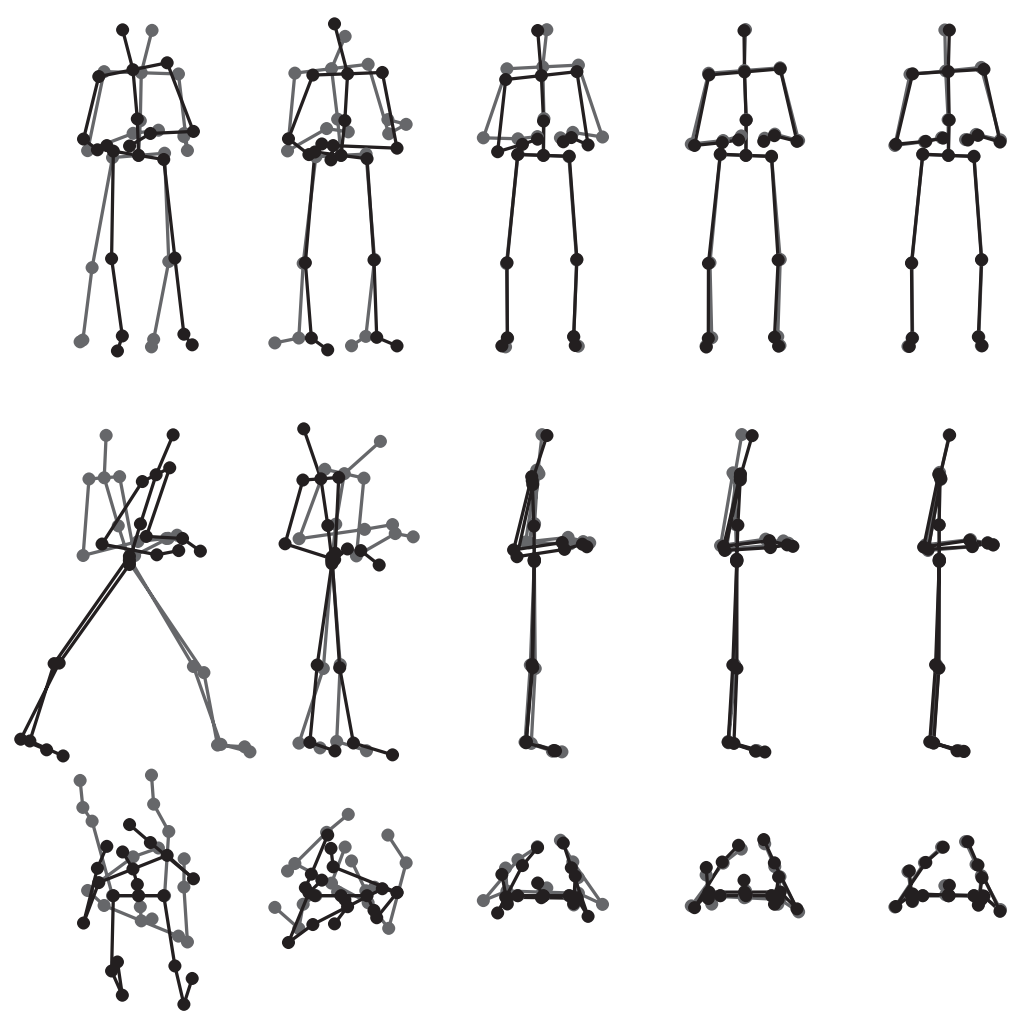

FIGURE 2. Movement complexity illustrated by displaying the projections of the first five principal components of complex movement (a) and noncomplex movement (b). The extreme deflections of the projections/dimensions are plotted from the front, from the side, and from the top. (a) High movement complexity, as movement is visible in all five PC projections, thus a high number of principal components is needed to explain the movement; (b) Low movement complexity, as most movement is found in the projection of PC 1-a low number of PCs can sufficiently explain the movement. 
TABLE 1. Results of the Intraclass Correlations for Both the Movement Features (a) and the Ratings of the Emotional Contents (b).

(a)

\begin{tabular}{lcllc}
\hline Movement Feature & $r$ & & Emotion & $r$ \\
\hline Torso Tilt & $.86^{\star *}$ & Arousal & $.98^{\star *}$ \\
Hand Distance & $.62^{\star *}$ & & Valence & $.94^{\star *}$ \\
Foot Distance & $.73^{\star *}$ & Happiness & $.95^{\star *}$ \\
Head Acceleration & $.95^{\star *}$ & Anger & $.97^{\star *}$ \\
Hand Acceleration & $.94^{\star *}$ & Sadness & $.92^{\star *}$ \\
Foot Acceleration & $.94^{\star *}$ & Tenderness & $.95^{\star *}$ \\
Area of Movement & $.38^{\star}$ & & \\
Fluidity & $.96^{\star *}$ & & \\
Rotation Range & $.60^{\star *}$ & & \\
Movement Complexity & $.77^{\star *}$ & & \\
\hline
\end{tabular}

${ }^{*} p<.05,{ }^{* *} p<.0001$

\section{Results}

In order to perform further analysis, we first assessed interparticipant consistency by calculating intraclass correlations (cf. Shrout \& Fleiss, 1979) for both the movement features and the ratings of the emotional contents. The values are displayed in Table 1. These values show that all movement features and emotion ratings received significant correlation values. Thus, the interparticipant consistency was sufficiently high for subsequent averaging of movement features and emotion ratings across participants to receive one value per stimulus.

In the first part of the analysis, the six rating items were correlated with the ten movement features to investigate the relations between the emotion ratings and the music-induced movement features. Due to the large number of correlations (60), we utilized false discovery rate control by applying the Benjamini-Hochberg procedure (see Benjamini \& Hochberg, 1995). At level $p=.05,17$ correlations were retained, with a largest $p$-value of .01. The results are shown in Table 2 .

For the group of Postural Features, Torso Tilt correlated significantly with characteristics of the emotional content: negatively with Arousal, $r(30)=-.70, p<.001$, and positively with Tenderness, $r(30)=.58, p<.001$. Additionally, Foot Distance correlated negatively with Happiness, $r(30)=-.45, p<.05$. These results suggest that the participants had a more upright torso when the music was considered active, and were more bent forward during tender music, and that they had a smaller foot distance when the music was happy.

All three Acceleration Features (Local Features group) showed high positive correlations with Arousal: Head Acceleration: $r(30)=.75, p<.001$; Hand Acceleration: $r(30)=.76, p<.001$; Foot Acceleration: $r(30)=.68$, $p<.001$. Additionally, Head and Hand Acceleration showed significant negative correlations with Tenderness: Head Acceleration: $r(30)=-.63, p<.001$; Hand Acceleration: $r(30)=-.47, p<.01$. Thus, participants tended to use more accelerated movements of the whole body for active music, and less acceleration of the upper body when the music was considered tender.

Three of the Global Features-Fluidity, Rotation Range, and Movement Complexity-correlated significantly with the emotional content: Fluidity exhibited negative correlations with Arousal, $r(30)=-.84, p<.001$, and Anger, $r(30)=-.47, p<.01$, and positive correlations with Valence, $r(30)=.45, p<.05$, and Tenderness, $r(30)=.66, p<.001$, suggesting that participants moved in a fluid way with pleasant and tender music, but more irregularly when the music had an active or angry character. Rotation Range, in addition, correlated positively with Valence, $r(30)=.55, p<.01$, and Happiness, $r(30)=.55, p<.01$, and negatively with Anger, $r(30)=$ $-.47, p<.01$, suggesting that participants rotated their body to pleasant and happy music, whereas they performed less body rotation with music that expressed

TABLE 2. Results of the Correlation Between the Movement Features and the Rating Items.

\begin{tabular}{|c|c|c|c|c|c|c|}
\hline & Arousal & Valence & Happiness & Anger & Sadness & Tenderness \\
\hline TorsoTilt & $-.70^{\star * *}$ & .29 & -.13 & -.32 & .39 & $.58^{\star *}$ \\
\hline HandDist & .07 & .22 & .15 & -.32 & .08 & .11 \\
\hline FootDist & -.31 & -.38 & $-.45^{\star}$ & .34 & .38 & -.23 \\
\hline HeadAcc & $.75^{\star * *}$ & -.36 & .10 & .37 & -.30 & $-.63^{* *}$ \\
\hline HandAcc & $.76^{* * *}$ & -.18 & .25 & .15 & -.40 & $-.47^{\star}$ \\
\hline FootAcc & $.68^{\star * *}$ & .02 & .41 & -.04 & -.43 & -.28 \\
\hline MoveArea & .30 & .31 & .40 & -.19 & -.33 & .11 \\
\hline Fluidity & $-.84^{\star * *}$ & $.45^{\star}$ & -.07 & $-.47^{\star}$ & .39 & $.66^{* * *}$ \\
\hline RotRange & .14 & $.55^{\star *}$ & $.55^{\star \star}$ & $-.47^{\star}$ & -.27 & .38 \\
\hline MoveComp & .08 & .42 & $.54^{*}$ & -.42 & $-.58^{\star *}$ & .19 \\
\hline
\end{tabular}

${ }^{*} p<.05,{ }^{* *} p<.01,{ }^{* * *} p<.001$ 
anger. Movement Complexity showed a significant positive correlation with Happiness, $r(30)=.54, p<.01$, and a significant negative correlation with Sadness, $r(30)=$ $-.58, p<.001$. Thus, participants tended to use complex movements for happy music, and simpler movements for music that expressed sadness.

Besides the (discrete) emotion ratings, we were interested in the embodiments and relationships between movement features and basic emotion ratings in a two-dimensional space based on the valence and arousal ratings. Thus, the second part of the analysis was motivated by Russell's (1980) circumplex models of affect and the positioning of discrete emotions as polar coordinates in a space consisting of valence and arousal dimensions. Russell asked participants to sort 28 emotional terms (such as happy, excited, angry, or bored) in different ways and created five circular models by computing polar coordinates for each of the 28 terms based on different sorting approaches and scaling methods. These polar coordinates started from the origin (of the circular space) and were specified by the angle and the length based on the sorter agreement.

As an initial step, we developed an angular variable that we called Circular Affect, derived from the Valence and the Arousal ratings of each stimulus. This was carried out by first representing the two ratings for Valence and Arousal for each stimulus as a vector in a twodimensional space (the $\mathrm{x}$-coordinate represents the Valence rating, the y-coordinate represents the Arousal rating). A vector projection at any possible polar angle was then applied to this vector, to receive a value for every angle. The outcome of this approach, the Circular Affect variable, is displayed in Figure 3a. A mathematical description of the derivation is given in Appendix B.

Subsequently, Circular Affect was correlated with the ten movement features and with the four basic emotion ratings. As the last step, the maximal correlation values and the corresponding angles were obtained. This procedure is exemplified in Figure 3b, showing the correlation results of one movement feature (Foot Acceleration) with Circular Affect.

Figure 4 displays the maximal correlation values and the respective polar angles for the ten movement features in a two-dimensional space in relation to Arousal and Valence. Table 3 shows the same data numerically. Due to the definition of Circular Affect (see Appendix 2), the minimal correlations are obtained by reflecting the respective projection vectors about the origin (i.e., rotating by $180^{\circ}$ ). The respective angles of the minimal correlations are given in brackets, as they will offer further insights into relationships between our results and Russell's (1980) circumplex models.
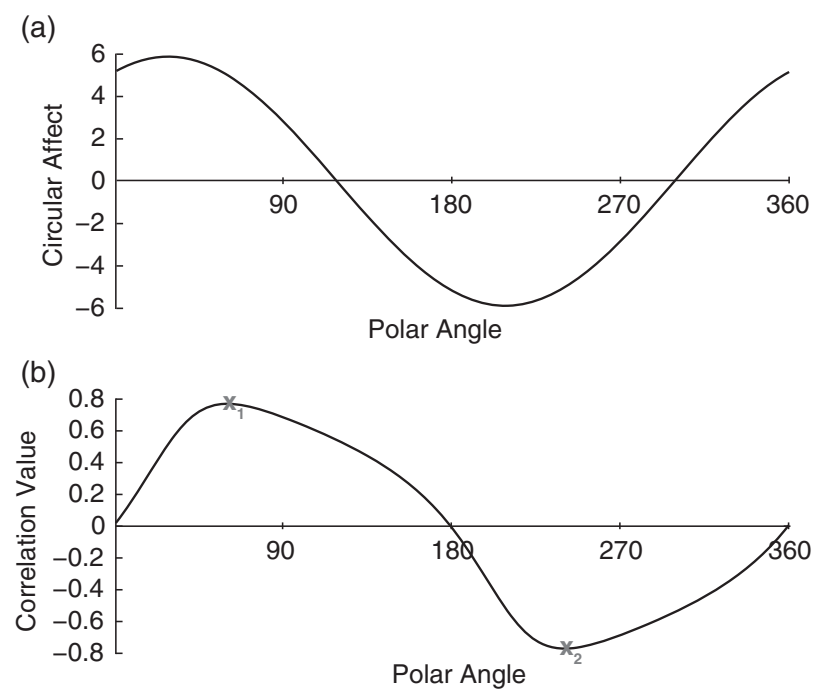

FIGURE 3. (a) Display of Circular Affect, i.e., the polar projection of the (averaged) rating for Valence and Arousal of one stimulus plotted as the function of the polar angle. (b) Correlation values between Circular Affect and Foot Acceleration plotted as the function of the polar angle. $X_{1}$ depicts the location of the maximal correlation, whereas $X_{2}$ depicts the location of the minimal correlation.

As a first step into analyzing the results, we would like to point out relationships between these results and the results of the previous analysis (discrete ratings, Table 2). Movement features for which the direction of the maximal correlation is close to the Arousal axis, such as Torso Tilt and Head Acceleration (cf. Figure 4), showed, in the discrete rating analysis (cf. Table 2), high correlations for Arousal, but low ones for Valence, whereas features located between the axes (e.g., around $45^{\circ}$ or $225^{\circ}$ ), such as Area of Movement or Foot Distance (while being significant in this analysis), exhibited low correlations for both discrete Valence and Arousal ratings (cf. Table 3).

It can be seen from Table 3/Figure 4 that there are a large number of high correlations between the movement features and Circular Affect. For the postural group, the correlations of Circular Affect with both Torso Tilt and Foot Distance were significant, with the maximal correlation value at an angle of $268^{\circ}$ (minimal correlation angle: $\left.88^{\circ}\right)(|r(30)|=.71, p<.001)$, respectively, for Torso Tilt, and at an angle of $217^{\circ}$ (min. corr.: $\left.37^{\circ}\right)(|r(30)|=.65, p<.001)$ for Foot Distance. These results suggest that participants had the most forward directed torso when the music was considered inactive, and the most upward directed torso when the music was considered active. Foot distance was biggest when the music was inactive and unpleasant, and smallest when the music was active and pleasant. 


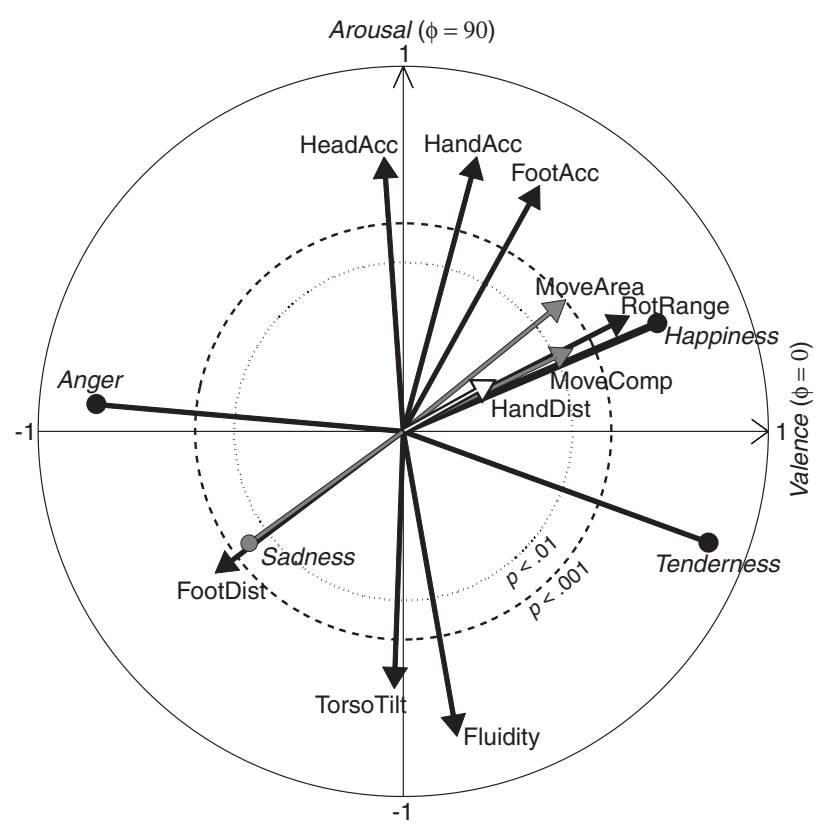

FIGURE 4. Maximal values of the correlations between Circular Affect and the ten movement features and the four basic emotion ratings respectively arranged in a circular model that we called Circular Affect Space (see Table 3 for values and angles in numerical form). The length of the arrows reflects the maximal value of the correlation, and the polar angle indicates the direction of the maximal value. Black arrowheads indicate a significance level of $p<.001$, grey arrowheads of $p<.01$, and white arrowheads are not significant. Movement features are indicated by sharp arrowheads, while the basic emotion ratings are indicated by rounded arrowheads.

All three Acceleration features correlated significantly with Circular Affect, with maximal values at $94^{\circ}$ (min. corr.: $\left.274^{\circ}\right)(|r(30)|=.75, p<.001)$ for Head Acceleration, at $75^{\circ}$ (min. corr.: $\left.255^{\circ}\right)(|r(30)|=.78, p<.001)$ for Hand Acceleration, and at $61^{\circ}$ (min. corr.: $241^{\circ}$ ) $(|r(30)|=.77, p<.001)$ for Foot Acceleration. Thus, the feet displayed highest acceleration when the music was active and pleasant, and lowest during music of the opposite character, whereas high hand and head acceleration was mostly observed when the music was active with neutral valence, and low hand and head acceleration with inactive music.

For the correlations of the Global Features with Circular Affect, the maximal values were also significant. For Area of Movement, it fell at an angle of $42^{\circ}$ (min. corr.: $\left.222^{\circ}\right)(|r(30)|=.64, p<.001)$, for Fluidity at an angle of $280^{\circ}$ (min. corr.: $\left.100^{\circ}\right)(|r(30)|=.85, p<.001)$, and for Rotation Range at an angle of $27^{\circ}$ (min. corr.: $\left.207^{\circ}\right)(|r(30)|=.70, p<.001)$. This outcome suggests that participants covered the largest area with pleasant and active music and the smallest area when the music
TABLE 3. Maximal Values and Corresponding Polar Angles in the Circular Affect Space of the Correlations Between Circular Affect and the Ten Movement Features as well as Between Circular Affect and the Basic Emotion Ratings.

\begin{tabular}{|c|c|c|}
\hline $\begin{array}{l}\text { Movement Feature/Basic } \\
\text { Emotion Rating }\end{array}$ & Correlation & $\begin{array}{l}\text { Max. Corr. } \\
\text { Angle }\end{array}$ \\
\hline Torso Tilt & $.71^{* * *}$ & $268^{\circ}$ \\
\hline Hand Distance & .29 & $29^{\circ}$ \\
\hline Foot Distance & $.65^{\star * *}$ & $217^{\circ}$ \\
\hline Head Acceleration & $.75^{\star * *}$ & $94^{\circ}$ \\
\hline Hand Acceleration & $.78^{\star * *}$ & $75^{\circ}$ \\
\hline Foot Acceleration & $.77^{* * *}$ & $61^{\circ}$ \\
\hline Area of Movement & $.57^{\star *}$ & $39^{\circ}$ \\
\hline Fluidity & $.85^{\star * *}$ & $280^{\circ}$ \\
\hline Rotation Range & $.70^{\star * *}$ & $27^{\circ}$ \\
\hline Movement Complexity & $.52^{\star \star}$ & $26^{\circ}$ \\
\hline Happiness & $.80^{* * *}$ & $23^{\circ}$ \\
\hline Anger & $.88^{\star * *}$ & $175^{\circ}$ \\
\hline Sadness & $.56^{\star *}$ & $216^{\circ}$ \\
\hline Tenderness & $.93^{\star * *}$ & $340^{\circ}$ \\
\hline
\end{tabular}

${ }^{* *} p<.01,{ }^{* * *} p<.001$

Note: We controlled the false discovery rate by using the Benjamini-Hochberg procedure.

was unpleasant and inactive. Participants were most fluid in their movement when the music was inactive and slightly pleasant, and least fluid with active and slightly unpleasant music. Furthermore, participants tended to rotate their body most for pleasant and slightly active music, and least for unpleasant and inactive music. Furthermore, Movement Complexity was found to have the maximal value of the correlation with Circular Affect at $26^{\circ}$ (min. corr.: $\left.206^{\circ}\right)(|r(30)|=.52, p<.01)$. Thus, participants tended to use complex movement for pleasant and slightly active music, and simple movement for unpleasant and slightly inactive music.

Additionally, the basic emotion ratings were correlated with Circular Affect to investigate the relationship between the different ratings. The results are depicted in Figure 4 as well. All four maximal correlation values were significant. For Happiness, the maximal value was at $23^{\circ}, r(30)=.80, p<.001$, for Anger at $175^{\circ}, r(30)=$ $.88, p<.001$, for Sadness at $216^{\circ}, r(30)=.56, p<.01$, and for Tenderness at $340^{\circ}, r(30)=.93, p<.001$.

\section{Discussion}

The results of this study suggest relationships between the emotional content of music and characteristics of music-induced movement. Each emotion rating correlated significantly with a different set of movement features that could therefore be assumed to be emotionspecific. 
Furthermore, the results provide support for Leman's (2007) concepts Embodied Attuning and Empathy. Movement features and perceived emotional content of music were found to correlate, so participants might have (unconsciously) used their body to express and reflect the affective content. However, the emotional content is created and shaped by features of the music, such as rhythm, timbre, or tonality-characteristics that could also be linked to the concept of Embodied Attuning. Previous research showed that participants rated the perceived emotional content of music consistently (Eerola \& Vuoskoski, 2011), and in order to change the emotional characteristics, musical features have to be changed. Thus, it may be difficult to distinguish between Embodied Attuning and Empathy, suggesting that they are overlapping and coexisting. However, since music can express and convey emotional characteristics, one could assume that the affective content of music has an unconscious effect on music-induced movement and suggests understanding Empathy as an abstraction of Embodied Attuning.

Since previous studies followed different purposes and used different techniques and a wide variety of movement descriptors that partly differ from ours, the comparison of the results is difficult. However, the results of our study still follow general directions proposed in previous studies. Our findings are quite in line with those by Camurri et al. (2003), Boone and Cunningham (1998), and Dahl and Friberg (2007) in that the quasi-spontaneous music-induced movements exhibited qualities similar to movements of professional dancers who embedded different emotional expressions in their dancing, and also to movements of instrumentalists who conveyed certain emotions while playing. Furthermore, our results confirm, at least partly, the hypothesized similarities to studies on emotionspecific nonverbal behavior, in particular to findings by De Meijer (1989), Darwin (1872), or Wallbott (1998). Finally, our results were consistent with the locations of emotional terms in Russell's (1980) circumplex models.

Basic emotions. Happiness correlated positively with Rotation Range and Movement Complexity, suggesting that happy music was expressed by using body rotation and high-dimensional movements. Although these particular movement features were not part of previous research, they might be related to features that were used in other studies. For example, the descriptions by Camurri et al. (2003) (dynamic movement, changes between low and high tension, and frequent tempo changes with long stops) or by De Meijer (1989) (fast, active, open, light, upward directed) could result in complex movement. In previous research, movements expressing happiness were found to be related to movements of the hands and arms (e.g., Boone \& Cunningham, 1998; Dael et al., 2012). Although, in the present analysis, hand acceleration did not significantly correlate with the happiness ratings, hand and especially arm movements can be expected to increase the complexity of movements, as the arms offer the best possibilities to move freely in different directions and independently from other body parts. Moreover, the relationship between Rotation Range and Happiness is supported by Burger and Bresin (2010), whose robot conveyed happiness by performing circular movements. Happiness also correlated negatively with Foot Distance. A smaller distance between the feet could be a result of increased movement complexity and rotation for happy music, as the feet are more active and potentially closer together (and even crossing in case of rotating around the vertical axis).

Anger was highly negatively correlated with Fluidity and Rotation Range, indicating that participants, when being exposed to music that is considered angry, were moving in an irregular and jagged way while not rotating the body. The co-occurrence of non-fluid movements and anger is well supported by the literature: Dahl and Friberg (2007) mentioned low fluency, Camurri et al. (2003) frequent tempo changes and short movements, Boone and Cunningham (1998) directional and tempo changes, and Darwin (1872) trembling. Jerkiness and non-fluency of movement might explain the negative correlation between Rotation Range and Anger, as smooth rather than jerky movements are associated with full rotations of the body.

The significant negative correlation between Sadness and Movement Complexity suggests that sad music is reflected in rather simple movements of low dimensionality. This result seems straightforward and is also supported by previous research. For example, Dahl and Friberg (2007) referred to low amount and speed of movement, Camurri et al. (2003) to low tension, few changes, long and smooth movements, and Wallbott (1998) to inactive movements covering little space-all characteristics that indicate movement of low complexity.

Tenderness was found to exhibit significant positive correlations with Torso Tilt and Fluidity and significant negative correlations with Acceleration of Head and Hands, indicating that tender music was embodied through a forward tilted torso and fluid movements with low acceleration. These movement characteristics would certainly fit to tender music, as tender music 
might not consist of strong and easily perceivable beats. Additionally, a forward tilted body, opposite to an upright torso showing alertness, could emphasize the relaxed character of tender music.

Dimensional model. Variables of the dimensional model have rarely been used in this field of research so far. However, active music was characterized by a high amount of movement and other movements that show the activity in the music, such as high acceleration of head, hands and feet, non-fluent movement, and an upright torso. In previous research (Boone \& Cunningham, 1998; Camurri et al., 2003; Dahl \& Friberg, 2007; Wallbott, 1998), acceleration-related movement features were associated with the expression of anger and happiness, both considered active emotions. Thus, it would be straightforward to assume that active music is reflected by similar movement characteristics. Moreover, an upright torso was found to be related to happiness and anger (Darwin, 1872; Wallbott, 1998), though De Meijer (1989) and Coulson (2004) reported an upward directed torso for happiness, but a bowed torso for angry. However, this discrepancy might suggest torso tilt to be a feature that discriminates pleasant and unpleasant active emotions.

The high negative correlation of Arousal with Fluidity is somewhat surprising, especially because the correlation value for Anger is lower than for Arousal. Based on related research, one could assume angry music to cause the highest amount of non-fluent movement (Boone \& Cunningham, 1998; Dahl \& Friberg, 2007), though our results might suggest non-fluid movements to be more characteristic for active than for angry music, at least in our set of stimuli.

Interesting to note is that Tenderness and Arousal exhibit a reverse correlation pattern. This suggests that Arousal and Tenderness might share the same movement features, but with opposite characteristics. The relation of both emotions will be discussed again in the following section.

Fluidity and Rotation Range were the only two significant movement characteristics for pleasant music, thus participants moved smoother and were rotating their body remarkably more when being exposed to pleasant music. Rotation Range was also found to be significant for happy music, thus a pleasant emotional character in music in general could be associated with body rotation.

Circular affect and the circular affect space. The Circular Affect Space indicates the locations of the maximal and minimal correlations between Circular Affect and the movement features, and subsequently reveals interesting insights into the embodiments of the emotion ratings related to the dimensional model. The polar angles corresponding to the maximal correlations are dispersed within the Circular Affect Space with an accumulation in the pleasant active quadrant (cf. Figure 4). Movement features related to amount and complexity of movement, such as Movement Complexity, Area of Movement, or Acceleration, can be found there, thus music perceived as pleasant and active might be encouraging to dance or move to. Likewise, the minimal correlations would cluster in the unpleasant inactive quadrant, suggesting that unpleasant and inactive music decreases the complexity and amount of movement due to the fact that such music is not as inviting to move along with.

Movement Complexity and Rotation Range resulted in similar angles $\left(26^{\circ}\right.$ and $\left.27^{\circ}\right)$ for the maximal correlation, which could suggest that they form a characteristic pattern for pleasant, slightly active music. Additionally, these two movement features were found to correlate significantly with the ratings for Happiness, which would indicate Happiness to be located at around $26 / 27^{\circ}$ in the Circular Affect Space in terms of the embodiment of musical emotions. This suggestion is supported by the correlation results between the happiness ratings and Circular Affect, which positions the maximal correlation at an angle of $23^{\circ}$.

Furthermore, Rotation Range was found to be related to Valence in the dimensional model analysis. Thus, body rotation seems to be especially characteristic for pleasant music.

In the basic emotions analysis, anger ratings correlated negatively with Fluidity and Rotation Range. The minimal correlation of Fluidity and Circular Affect falls into the (upper) unpleasant active quadrant, whereas the minimal correlation of Rotation Range and Circular Affect falls into the (upper) unpleasant inactive quadrant. The relatively low correlation values of both features with the anger ratings $(r=-.47)$ could motivate the conclusion that the middle of both minimal correlations (around $150^{\circ}$ ) is the estimated location for Anger in the Circular Affect Space regarding the embodiment of musical emotions. This estimated location is relatively close to the position of the maximal correlation value of the anger ratings with Circular Affect at $175^{\circ}$, which would support the consistency between the discrete ratings to some extent. However, a more active position could have been expected, since anger is usually related to both (positive) arousal and (negative) valence, so this result is somewhat surprising.

The minimal correlations that lie in the unpleasant inactive quadrant-especially Movement Complexitycould be linked to Sadness. Low Movement Complexity 
was found to be characteristic for sad music in the basic emotions analysis, suggesting that Sadness might be located at around $205^{\circ}$ in the Circular Affect Space. This angle might appear as "too active," though it is rather difficult to find genuinely sad stimuli that encourage movement. The correlation between the sadness ratings and Circular Affect resulted in a maximal value located at $216^{\circ}$, which would be consistent with the suggested location mentioned above. Interesting to note is that the location of Sadness is very similar to the location of Foot Distance, although Foot Distance failed to significantly correlate with Sadness in the discrete emotion analysis. Since Foot Distance was not part of previous studies, it is rather difficult to give suitable explanations here. A possible explanation could nonetheless be that participants adopted more stable footing with feet spaced wider apart, since sad music also led participants to move less (cf. the basic emotion rating results discussed above).

By observing the minimal correlations, the characteristic movement pattern for tender music could be linked to this analysis as well. Head and Hand Acceleration received significant negative correlations with the ratings for Tenderness, thus it could be expected that the minimal correlation with these two movement features would be located in the Tenderness (or pleasant inactive) quadrant. However, they are close to the arousal axis, and thus more related to inactive neutral stimuli. It could therefore be suggested that Tenderness might be located quite close to the Arousal axis, which would explain the opposite correlation pattern of Tenderness and Arousal. The lower correlation value for Hand Acceleration $(r=-.47)$ and the higher value for Head Acceleration $(r=-.63)$ could lead to the conclusion that Tenderness might be located in the pleasant inactive quadrant somewhat close to the negative Arousal axis. The maximal correlations of (forward) Torso Tilt and Fluidity would also support the suggested location of Tenderness. The location of the maximal correlation value of the tenderness ratings and Circular Affect $\left(340^{\circ}\right)$, however, is closer to the Valence axis than to the Arousal axis. Thus, this result is somewhat surprising, but maybe just reflects the nature of the stimuli being rather active in order to stimulate movement.

Maximal Fluidity, Torso Tilt, and minimal Hand, Head, and Feet Acceleration fall into the same region of the Circular Affect Space between Tenderness and Inactivity, thus participants were probably not able to distinguish between the two emotional states, which could furthermore explain the opposite correlation pattern of Tenderness and Arousal.

One movement feature, Area of Movement, that failed to be significant in the basic emotions analysis, was found to be significant in the Circular Affect analysis. The maximal correlation of Area of Movement with Circular Affect falls into the pleasant active quadrant; thus, listeners tend to cover a large area when moving to pleasant and active music. This finding could be linked to research by Wallbott (1998), who noted that movements displaying active emotions, such as happiness and anger, covered more space than movements displaying sadness, probably because sad music rather inhibits than encourages movement.

The circular affect space in relation to Russell's (1980) circumplex models. In what follows, we will compare and link the Circular Affect Space to Russell's (1980) circumplex models. We will refer here to the two models that were derived by direct circular scaling and by multidimensional scaling. Table 4 displays the emotions of Russell's circumplex models that are most proximate to the locations of the maximal and minimal correlations between movement features and Circular Affect.

In case of the direct circular scaling, Russell's 'delighted' $\left(25^{\circ}\right)$ is most closely located to Movement Complexity, Rotation Range, and Hand Distance, whereas 'happy' $\left(8^{\circ}\right)$ is located too low on the arousal axis to match any of our movement feature correlations. However, both emotions are positive, thus they could be related to similar movement features. The location of the maximal correlation between Circular Affect and Area of Movement is closest to 'excited' $\left(49^{\circ}\right)$, indicating that participants cover a bigger area when moving to exciting and activating music. The location of 'aroused' $\left(74^{\circ}\right)$ in the upper pleasant active quadrant fits with Acceleration of Feet and Hands, which supports our basic emotion/dimensional model analysis. However, Head Acceleration is closest to 'alarmed' $\left(96^{\circ}\right)$, and also close to 'angry' $\left(99^{\circ}\right)$, which did not correlate in our basic emotions analysis. Foot Distance is located close to 'depressed' $\left(210^{\circ}\right)$ and 'sad' $\left(208^{\circ}\right)$, suggesting that a large distance between feet could be related to unpleasant inactive music. Torso Tilt can be found close to 'tired' $\left(268^{\circ}\right)$, which is straightforward, as one bends forward when being tired. Fluidity is closest located to 'sleepy' $\left(272^{\circ}\right)$, which suggests inactive neutrally pleasant stimuli to be embodied with fluid movements.

The angles corresponding to the minimal correlations for Circular Affect with Rotation Range, Movement Complexity, and Hand Distance, are close to Russell's location of 'sad,' which supports the above suggested location for sadness in the Circular Affect Space and furthermore the finding that sad music can be related to low complexity of movement. Area of Movement is close to 'depressed' and ', suggesting that unpleasant 
TABLE 4. Emotions of Russell's (1980) Circumplex Models that are Closest Located to the Maximal and Minimal Correlations Between Circular Affect and the Movement Features.

\begin{tabular}{|c|c|c|c|c|}
\hline \multirow{2}{*}{ Movement feature } & \multicolumn{2}{|c|}{$\begin{array}{l}\text { Russell Emotion Corresponding to } \\
\text { Maximal Correlation Direction }\end{array}$} & \multicolumn{2}{|c|}{$\begin{array}{l}\text { Russell Emotion Corresponding to } \\
\text { Minimal Correlation Direction }\end{array}$} \\
\hline & Direct Scaling & Multidimensional Scaling & Direct Scaling & Multidimensional Scaling \\
\hline TorsoTilt & Tired & sleepy & tense & aroused \\
\hline HandDist & Delighted & happy & sad & depressed \\
\hline FootDist & Depressed & sad & delighted & delighted \\
\hline HeadAcc & Alarmed & aroused & sleepy & sleepy \\
\hline HandAcc & Aroused & astonished & droopy & sleepy \\
\hline FootAcc & Astonished & excited & bored & tired \\
\hline MoveArea & Exited & delighted & depressed & bored \\
\hline Fluidity & Sleepy & sleepy & angry & aroused \\
\hline RotRange & Delighted & happy & sad & depressed \\
\hline MoveComp & Delighted & happy & sad & depressed \\
\hline
\end{tabular}

Note: The second and fourth columns of the table correspond to the circumplex model derived by direct circular scaling and the third and fifth columns to the circumplex model derived by multidimensional scaling.

inactive music is embodied with movements within a small area. The minimal correlations of Head, Hand, and Feet Acceleration are close to 'sleepy,' 'droopy' $\left(256^{\circ}\right)$, and 'bored' $\left(241^{\circ}\right)$, suggesting that inactive music is related to low acceleration of the body. Foot Distance is located between Russell's 'delighted' and 'excited,' indicating that small distance between feet might be characteristic for music with such emotional qualities. Torso Tilt is located close to 'tense' $\left(92^{\circ}\right)$, suggesting that tense music is embodied by an upward torso. The minimal correlation of Fluidity is close to the location of 'angry,' which would support both previous research and our basic emotions analysis.

In the case of the multidimensional scaling solution, 'happy' $\left(19^{\circ}\right)$ is located closest to the maximal correlations between Circular Affect and Movement Complexity and Rotation Range (and Hand Distance), and would therefore support our basic emotion analysis and reflect the location of Happiness in the Circular Affect Space. Area of Movement is close to 'delighted' $\left(44^{\circ}\right)$. Acceleration of Feet and Hands are near 'exited' $\left(72^{\circ}\right)$ and 'astonished' $\left(77^{\circ}\right)$, whereas Head Acceleration is closest to 'aroused' $\left(93^{\circ}\right)$, supporting our previous analyses. None of our maximal correlations match Russell's 'angry' $\left(140^{\circ}\right)$. Foot Distance seems again to be related to 'sad' $\left(214^{\circ}\right)$, and 'sleepy' $\left(271^{\circ}\right)$ is close to Torso Tilt and Fluidity.

The minimal correlations of Circular Affect and Rotation Range, Movement Conplexity, and Hand Distance are close to Russell's 'depressed' $\left(204^{\circ}\right)$, and Area of Movement is close to 'bored' $\left(225^{\circ}\right)$, while 'sad' $\left(214^{\circ}\right)$ is located between both emotions, suggesting that such unpleasant, inactive music is embodied with low complexity and simple movements using a small area. Acceleration of Feet, Hands, and Head are close to 'tired' $\left(242^{\circ}\right)$ and 'sleepy' $\left(271^{\circ}\right)$. Foot Distance is close to Russell's 'delighted,' whereas Torso Tilt and Fluidity are closest to 'aroused,' supporting that active music with neutral valence is embodied with an upright torso and non-fluid movements.

The locations of the maximal values of the correlations between the basic emotion ratings and Circular Affect are closer to the locations of Russell's multidimensional scaling solution than to his direct scaling solution. Both Happiness and Sadness obtained similar angles as in Russell's solution. Tenderness, not having a direct counterpart in Russell's analysis, is located close to Russell's 'serene' and 'calm,' suggesting their similarity. Russell's 'angry' can be found at a more active position than our correlational analysis has placed it, close to suggested location based on the correlation pattern between the anger ratings and the movement features.

\section{Conclusion}

This study offers insights into body-related nonverbal behavior, and indicates that there are relationships between the emotional content of music and musicinduced, quasi-spontaneous movement. Characteristic movements and movement combinations were associated with different emotional characteristics of music. It is thus argued that the body reflects emotional qualities of music, which can be seen as support for the notion of embodied music cognition, especially for the concept of Empathy and its potential boundaries to Embodied Attuning. Furthermore, a circular variable, Circular 
Affect, was derived from the arousal and valence ratings, and the locations of the maximal correlations between movement features and these compound ratings were displayed in a two-dimensional space. Both the results of the emotion ratings assessed in this study and Russell's circumplex model of affect show remarkable consistency with the Circular Affect model.

Previous research in the field tended to use professional actors, musicians, or dancers performing certain emotional states, with their attention focused on each emotion. It is likely that this approach results in exaggerated, artificial, and stylized movements. This study, on the other hand, focused on laypersons and investigated how the emotional content of music affects movement when participants are not actively and consciously paying attention to it. As a consequence, the resulting movements might be more diverse and difficult to analyze than movements performed by professional dancers. However, we believe that our approach is ecologically more valid than the acted emotions approaches for investigating the impact of emotional content of music on everyday behavior.

It could be argued that our participants moved rather according to musical features than according to the emotional content. However, in the present analysis approach, the role of musical features was not considered. Future work could therefore include extracting musical features and subsequently using them, for instance, in multiple regression models together with the movement features and the emotion ratings. This would give further insights into the relation between movement features, emotional content, and musical features, and also in possible distinction between Leman's (2007) concepts of Empathy and Embodied Attuning.

Finally, an emotion recognition experiment could be conducted in which participants are asked to rate the movements presented as point-light videos regarding the emotion conveyed or reflected. This would give insight into the perception of emotions in musicinduced, quasi-spontaneous movements.

\section{Author Note}

We thank Mikko Leimu for help and technical assistance during the motion capture data collection. We also wish to thank the three anonymous reviewers for their thorough and helpful comments on the manuscript. This study was funded by the Academy of Finland (projects 118616, 125710, and 136358).

Correspondence concerning this article should be addressed to Birgitta Burger, Department of Music, P.O. Box 35, FI-40014 University of Jyväskylä, Finland. E-mail: birgitta.burger@jyu.fi

\section{References}

$\rightarrow$ Balkwill, L.-L., \& Thompson, W. F. (1999). A cross-cultural investigation of the perception of emotion in music: Psychophysical and cultural cues. Music Perception, 17, 43-64.

$\rightarrow$ Benjamini, Y., \& Hochberg, Y. (1995). Controlling the false discovery rate: A practical and powerful approach to multiple testing. Journal of the Royal Statistical Society, Series B (Methodological), 57, 289-300.

$\rightarrow$ Boone, R. T., \& Cunningham, J. G. (1998). Children's decoding of emotion in expressive body movement: The development of cue attunement. Developmental Psychology, 34, 1007-1016.

$\rightarrow$ Burger, B., \& Bresin, R. (2010). Communication of musical expression by means of mobile robot gestures. Journal on Multimodal User Interfaces, 3, 109-118.

Burger, B., Thompson, M. R., Saarikallio, S., Luck, G., \& Toiviainen, P. (2010). Influence of musical features on characteristics of music-induced movements. In S. M. Demorest, S. J. Morrison, \& P. S. Campbell (Eds.), Proceedings of the 11th International Conference on Music Perception and Cognition (ICMPC) (pp. 425-428). Seattle, WA: University of Washington.

$\rightarrow$ Camurri, A., Lagerlöf, I., \& Volpe, G. (2003). Recognizing emotion from dance movement: Comparison of spectator recognition and automated techniques. International Journal of Human-Computer Studies, 59, 213-225.

Camurri, A., Mazzarino, B., Ricchetti, M., Timmers, R., \& Volpe, G. (2004). Multimodal analysis of expressive gesture in music and dance performances. In A. Camurri \& G. Volpe (Eds.), Gesture-based communication in human-computer interaction. Lecture notes in computer science, 2915 (pp. 20-39). Berlin/Heidelberg, Germany: Springer.

Castellano, G., Villalba, S. D., \& Camurri, A. (2007). Recognising human emotions from body movement and gesture dynamics. In A. Paiva, R. Prada, \& R. W. Picard (Eds.), Proceedings of the 2nd International Conference in Affective Computing and Intelligent Interaction (ACII) (pp. 71-82). Berlin/Heidelberg, Germany: Springer.

Clynes, M. (1980). The communication of emotion: Theory of sentics. In R. Plutchik, \& H. Kellerman (Eds.), Theories of emotions (Vol. 1, pp. 171-216). New York, NY: Academic Press.

Clynes, M. (1992). Time-forms, nature's generators and communicators of emotion. In Proceedings of the IEEE International Workshop on Robot and Human Communication (pp. 18-31), Tokyo, Japan. 
$\rightarrow$ Coulson, M. (2004). Attributing emotion to static body postures: Recognition accuracy, confusions, and viewpoint dependence. Journal of Nonverbal Behavior, 28, 117-139.

$\rightarrow$ Dael, N., Mortillaro, M., \& Scherer, K. R. (2012). Emotion expression in body action and posture. Emotion, 12, 1085-1101.

$\rightarrow$ Dahl, S., \& Friberg, A. (2007). Visual perception of expressiveness in musicians' body movements. Music Perception, 24, 433-454.

DARWIN, C. (1872). The expression of the emotions in man and animals. London, UK: John Murray.

$\rightarrow$ De Mejjer, M. (1989). The contribution of general features of body movement to the attribution of emotions. Journal of Nonverbal Behavior, 13, 247-268.

$\rightarrow$ Eerola, T., \& Vuoskoski, J. K. (2011). A comparison of the discrete and dimensional models of emotion in music. Psychology of Music, 39, 18-49.

Eкмал, P. (Ed.) (1982). Emotions in the human face. Cambridge, UK: Cambridge University Press.

$\rightarrow$ Eкman, P., \& Friesen, W. V. (1974). Detecting deception from the body or face. Journal of Personality and Social Psychology, 29, 288-298.

$\rightarrow$ Evans, P., \& Schubert, E. (2008). Relationships between expressed and felt emotions in music. Musicae Scientiae, 12, 75-99.

Gabrielsson, A. (2002). Emotion perceived and emotion felt: Same or different? Musicae Scientiae, 6, 123-147.

$\rightarrow$ Gabrielsson, A., \& Juslin, P. N. (1996). Emotional expression in music performance: Between the performer's intention and the listener's experience. Psychology of Music, 24, 68-91.

Gabrielsson, A., \& Lindström, E. (2001). The influence of musical structure on emotional expression. In P. N. Juslin \& J. A. Sloboda (Eds.), Music and emotion: Theory and Research (pp. 223-248). New York: Oxford University Press.

$\rightarrow$ Ilie, G., \& Thompson, W. F. (2006). A comparison of acoustic cues in music and speech for three dimensions of affect. Music Perception, 23, 319-330.

$\rightarrow$ Janata, P., Tomic, S. T., \& Haberman, J. M. (2011). Sensorimotor coupling in music and the psychology of the groove. Journal of Experimental Psychology: General, 141, 54-75.

$\rightarrow$ Keller, P., \& Rieger, M. (2009). Special issue: Musical movement and synchronization. Music Perception, 26, 397-400.

$\rightarrow$ Krumhansl, C. L. (2002). Music: A link between cognition and emotion. Current Directions in Psychological Science, 11, 45-50.

Lakoff, G., \& Johnson, M. (1980). Metaphors we live by. Chicago, IL: University of Chicago Press.

Lakoff, G., \& Johnson, M. (1999). Philosophy in the flesh: The embodied mind and its challenge to Western thought. New York: Basic Books.
Leman, M. (2007). Embodied music cognition and mediation technology. Cambridge, MA, London, UK: MIT Press.

Leman, M., \& Godøy, R. I. (2010). Why study musical gesture? In R. I. Godøy \& M. Leman (Eds.), Musical gestures. Sound, movement, and meaning (pp. 3-11). New York: Routledge.

$\rightarrow$ Lesaffre, M., De Voogdt, L., Leman, M., De Baets, B., De Meyer, H., \& Martens, J.-P. (2008). How potential users of music search and retrieval systems describe the semantic quality of music. Journal of the American Society for Information Science and Technology, 59, 695-707.

$\rightarrow$ Luck, G., Saarikallio, S., Burger, B., Thompson, M. R., \& Toiviainen, P. (2010). Effects of the Big Five and musical genre on music-induced movement. Journal of Research in Personality, 44, 714-720.

$\rightarrow$ Russell, J. A. (1980). A circumplex model of affect. Journal of Personality and Social Psychology, 39, 1161-1178.

$\rightarrow$ SaArikallio, S. (2011). Music as emotional self-regulation throughout adulthood. Psychology of Music, 39, 307-327.

$\rightarrow$ Savitzky, A., \& Golay, M. J. E. (1964). Smoothing and differentiation of data by simplified least squares procedures. Analytical Chemistry, 36, 1627-1639.

$\rightarrow$ Schlosberg, H. (1954). Three dimensions of emotion. Psychological Review, 61, 81-88.

$\rightarrow$ Schubert, E. (1999). Measuring emotion continuously: Validity and reliability of the two-dimensional emotion-space. Australian Journal of Psychology, 51, 154-165.

$\rightarrow$ Shrout, P. E., \& Fleiss, J. L. (1979). Intraclass correlations: Uses in assessing rater reliability. Psychological Bulletin, 86, 420-428.

Toiviainen, P., \& Burger, B. (2011). MoCap toolbox manual. Jyväskylä, Finland: University of Jyväskylä. Available at http:// www.jyu.fi/music/coe/materials/mocaptoolbox/MCTmanual.

Van Dyck, E., Moelants, D., Demey, M., Coussement, P., Deweppe, A., \& Leman, M. (2010). The impact of the bass drum on body movement in spontaneous dance. In S. M. Demorest, S. J. Morrison, \& P. S. Campbell (Eds.), Proceedings of the 11th International Conference in Music Perception and Cognition (ICMPC) (pp. 429-434), Seattle, WA: University of Washington.

Varela, F. J., Thompson, E., \& Rosch, E. (1991). The embodied mind: Cognitive science and human experience. Cambridge, MA: MIT Press.

$\rightarrow$ Walliotт, H. G. (1998). Bodily expression of emotion. European Journal of Social Psychology, 28, 879-896.

$\rightarrow$ Wanderley, M. M., Vines, B. W., Middleton, N., McKay, C., \& Натсн, W. (2005). The musical significance of clarinetists' ancillary gestures: An exploration of the field. Journal of New Music Research, 34, 97-113.

$\rightarrow$ Zentner, M., Grandjean, D., \& Scherer, K. R. (2008). Emotions evoked by the sound of music: Characterization, classification, and measurement. Emotion, 8, 494-521. 


\section{Appendix A: List of Stimuli}

Alice Deejay: Better Off Alone (Who Needs Guitars Anyway?) 2:40-2:54 (loop)

Andre Visior: Speed Up 1:15-1:45

Antibalas: Who is this America Dem Speak of Today? (Who Is This America?) 1:00-1:29

Arturo Sandoval: A Mis Abuelos (Danzon) 1:53-2:21

Baden Powell: Deixa (Personalidade) 1:11-1:41

Brad Mehldau: Wave/Mother Nature's Son (Largo) 0:00-0:29

Clifford Brown \& Max Roach: The Blues walk (Verve Jazz Masters, Vol. 44: Clifford Brown \& Max Roach) 2:01-2:31

Conjunto Imagen: Medley-Esencia de Guaguanco/ Sonero (Ayer, Hoy y Manana) 2:18-2:48

Dave Hillyard \& The Rocksteady 7: Hillyard Street (Playtime) 0:15-0:45

Dave Weckl: Mercy, Mercy, Mercy (Burning for Buddy) 0:10-0:40

Dave Weckl: Tower of Inspiration (Master Plan) 0:00-0:30

DJ Shadow: Napalm Brain/Scatter Brain (Endtroducing... ) 3:29-3:58

Gangster Politics: Gangster Politics (Guns \& Chicks) 1:00-1:29

Gigi D'Agostino: Blablabla (L'Amour Toujours) 0:00-0:30

Herbie Hancock: Watermelon man (Cantaloupe Island) 0:00-0.30

Horace Silver: The Natives Are Restless 0:00-0:29

In Flames: Scream (Come Clarity) 0:00-0:30

Jean Roch: Can You Feel it (Club Sounds Vol. 35) 0:33-1:01

Johanna Kurkela: Hetki hiljaa (Hetki hiljaa) 3:22-3:52

Juana Molina: Tres cosas (Tres Cosas) 0:00-0:30

Kings of Leon: Closer (Only by the Night) 3:17-3:47

Lenny Kravitz: Live (5) 3:02-3:30

Martha \& The Vandellas: Heat Wave (Heat Wave) 1:40-2:10

Maynard Ferguson: Fireshaker (Live From San Francisco) 0:00-0:28

MIA: 20 Dollar (Kala) 0:17-0:45

Nick Beat: Techno Disco 2:26-2:56

Panjabi MC: Mundian To Bach Ke (Legalised) 0:471:06 (loop)

Patrick Watson: Beijing (Wooden Arms) 2:30-2:59

The Rippingtons: Weekend in Monaco (Weekend in Monaco) 1:13-1:42

Yuri Buenaventura: Salsa (Salsa Movie Soundtrack) 2:17-2:45

\section{Appendix B: Derivation of Circular Affect}

In what follows, the derivation of the angular variable Circular Affect is explained in mathematical terms.

The combination of the two ratings for Valence and Arousal for any stimulus can be represented as an emotion vector in a two-dimensional space, e.g., $\mathbf{r}=(\mathrm{v}, \mathrm{a})$. In order to obtain a value at any possible polar angle $\theta$, this vector $\mathbf{r}$ was orthogonally projected on the unit vector $\mathbf{e}$ with the respective polar angle,

$$
\mathbf{e}_{\theta}=\mathbf{i} \cos \theta+\mathbf{j} \sin \theta, \theta \in[0,2 \pi],
$$

where $\mathbf{i}$ and $\mathbf{j}$ denote the unit vectors parallel to the horizontal and vertical axes, respectively. The vector projection itself was then accomplished by the dot product:

$$
\mathrm{C}_{\theta}=\mathbf{r} \cdot \mathbf{e}_{\theta} \text {. }
$$

The general principle for this projection is displayed in Figure 5.

Subsequently, Circular Affect $\left(\mathrm{C}_{\theta}\right)$ was correlated with each of the movement features $M_{i}$ (respectively each basic emotion rating), resulting in a series of correlation values. In the last step, the maximal correlation value and the corresponding angle $\theta$ were obtained from this series.

$$
\arg \max _{\theta}=\operatorname{corr}\left(\mathrm{C}_{\theta}, \mathrm{M}_{\mathrm{i}}\right) .
$$

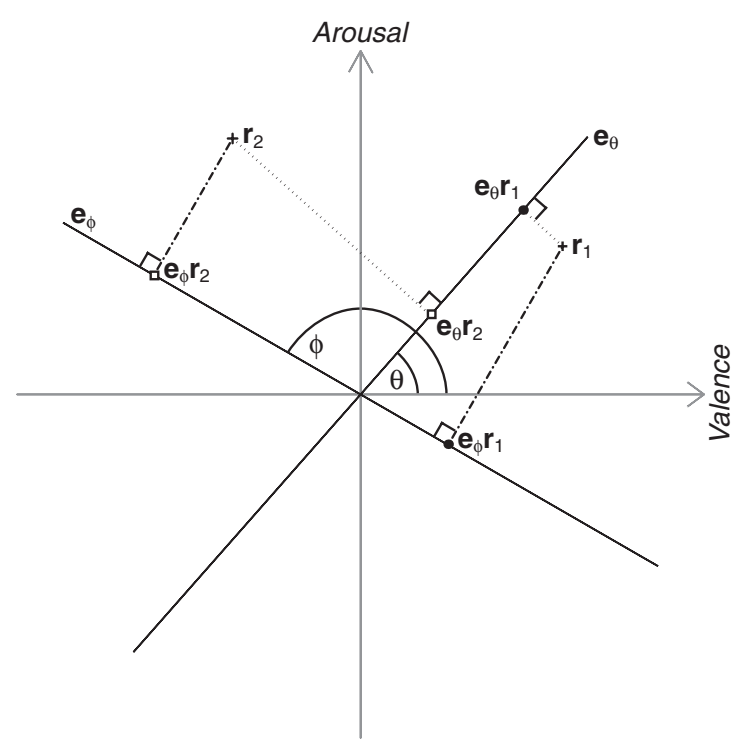

FIGURE 5. Derivation of the dimensional variable Circular Affect. $r_{1}$ is an arbitrary rating of Valence and Arousal for one stimulus, whereas $r_{2}$ is an arbitrary rating of Valence and Arousal for another stimulus. $e_{\theta}$ and $e_{\phi}$ are two arbitrary unit vectors at the polar angles $\theta$ and $\phi$. The vectors $r_{1}$ and $r_{2}$ are projected onto $e_{\theta}$ and $e_{\phi}$ and result in the values $e_{\theta} \cdot r_{1}$ and $e_{\phi} \cdot r_{1}$ of the Circular Affect variable for stimulus 1, and respectively in the values $e_{\theta} \cdot r_{2}$ and $e_{\phi} \cdot r_{2}$ for stimulus 2 . 